\title{
Effects of the rate of solvent evaporation on the characteristics of drug loaded PLLA and PDLLA microspheres
}

\author{
Tze-Wen Chung ${ }^{\text {a,* }}$, Yi-You Huang ${ }^{\text {b }}$, Yi-Ze Liu ${ }^{\text {a }}$ \\ a Department of Biomedical Engineering, Chung-Yuan Christian University, Chung-Li 32023, Taiwan, ROC \\ ${ }^{\mathrm{b}}$ Institute of Biomedical Engineering, National Taiwan University, Taipei, Taiwan, ROC
}

Received 23 March 2000; received in revised form 8 September 2000; accepted 8 September 2000

\begin{abstract}
We investigated the effects of the rate of solvent removal by varying ambient pressure at a fixed temperature on the morphology, particle sizes, drug encapsulation efficiency and releases pattern of lidocaine loaded poly-L-lactatide (PLLA) and poly-D,L-lactatide (PDLLA) microspheres, prepared with $\mathrm{O} / \mathrm{W}$ emulsion-solvent evaporation process. Prepared in the fast rate of solvent evaporation (FRSE) process by reducing ambient pressure, smoothly morphological surface of drug loaded PLLA and PDLLA microspheres was observed. While in the normal rate of solvent evaporation (NRSE) process, roughness or pinhole surface was only found at drug loaded PLLA microspheres. Fabricated in the FRSE process, both PLLA and PDLLA microspheres showed smaller particle sizes and lower drug encapsulation efficiencies than those prepared in NRSE process. In regard to two materials, PLLA microspheres had higher drug encapsulation efficiencies than PDLLA ones for both processes. Although initial burst releases of drug were observed for both PLLA and PDLLA microspheres prepared in whatever solvent removal process, drug release for PLLA microspheres was slightly less than that for PDLLA ones in the earlier stage of drug release. However, in the subsequent stage of drug release, there was no difference between two materials. In corporation with different crystalline characteristics of PLA polymer and its derivatives, FRSE process by reducing ambient pressure could be further applied to produce different characteristics of microspheres for drug delivery. (C) 2001 Elsevier Science B.V. All rights reserved.
\end{abstract}

Keywords: Poly-L-lactatide; Poly-D,L-lactatide; Solvent evaporation; Microspheres

\section{Introduction}

Poly-L-lactatide (PLLA), poly-D,L-lactatide (PDLLA) or their derivatives such as poly-D,Llactatide-co-glycolide (PLGA) have been widely applied as one of carriers for drug delivery. Since they are biodegradable and shown not to cause adverse tissue reactions (Conti et al., 1992;

\footnotetext{
* Corresponding author. Tel.: + 886-3-4563171, ext. 4108; fax: + 886-3-4563160.

E-mail address: twchung@medical.be.cycu.edu.tw (T.-W.
} Chung). 
Gogolewski et al., 1993; Gupta et al., 1997), there is no needed to retrieve or retreat the carrier after the drug is depleted.

The characteristics of drug loaded PLLA, PDLLA or PLGA microspheres are affected by process parameters such as the concentrations of drug, the volume ratios of disperse to continuous phase, and the technique of solvent removal (Sato et al., 1988; Arshady, 1991; Li et al., 1996; Atkins et al., 1998). In preparing PLLA or PDLLA microspheres loaded with hydrophobic drugs such as progesterone and lidocaine, dichloromethane solution (DCM), and polyvinyl alcohol solution (PVA) have been widely used as a solvent and stabilizer of the suspension, respectively (Cowsar et al., 1985; Izumikawa, 1991; Le Corre et al., 1994; Huang et al., 1999). After droplet suspension was formed, the techniques of solvent removal (e.g. temperature gradient or dilution of continuous phase) affected the characteristics of drug loaded PLLA or other PLA based microspheres (Izumikawa, 1991; Li et al., 1996; Huang et al., 1997; Jeyanthi et al., 1997; Yang et al., 2000). Among different solvent removal techniques, single step solvent evaporation has been used by many investigators (Cowsar et al., 1985; Izumikawa, 1991; Esposito et al., 1997; Huang et al., 1997). Reducing ambient pressure to facilitate solvent evaporation or so-called 'fast rate of solvent evaporation' (FRSE) process influenced the morphology and crystalline structure for progesterone loaded PLLA microspheres prepared in mechanical stirring-solvent evaporation process (Izumikawa, 1991). Whether FRSE process would also affect other characteristics of drug loaded PLLA or PDLLA microspheres, prepared by $\mathrm{O} / \mathrm{W}$ emulsion-solvent evaporation process, have not been investigated. We thereby investigated the effect FRSE technique by reducing ambient pressure at a fixed temperature on the characteristics of PLA based microspheres with lidocaine as a model drug.

\section{Materials and methods}

\subsection{Materials}

PLLA (Lot 92H0795) and PDLLA (Lot
117H0889) with an average molecular weights of 93 and $106 \mathrm{kDa}$, respectively, were purchased from Sigma Company. Lidocaine (Mw. 204) and PVA (degree of polymerization of 2000) were purchased from Nacclai Corp. (Nacclai tesque, Kyoto, Japan). DCM, chloroform and other reagents (Alps Chemical Corp., Hsinchu, Taiwan) were LC grade and used as received.

\subsection{Fabrication of PLLA and PDLLA microspheres with FRSE or NRSE process}

Emulsion-solvent evaporation method was applied to fabricate PLLA and PDLLA microspheres. The fabricating processes were similar to our previous report (Huang et al., 1999) except in solvent evaporating procedures. In general, $0.5 \mathrm{~g}$ of PLLA or $0.5 \mathrm{~g}$ of PDLLA and $0.05 \mathrm{~g}$ of lidocaine were dissolved in $5 \mathrm{ml}$ of DCM. After completely dissolved, it was poured into $50 \mathrm{ml}$ of $0.05 \mathrm{wt} . \%$ of PVA solution, an emulsifier, and then the solution was emulsified by a probe ultra-sonicator (GE 50T, Cole-Palmer Co., USA) under $4^{\circ} \mathrm{C}$ to form an $\mathrm{O} / \mathrm{W}$ emulsion.

The emulsified suspension was then mechanically stirred, and the solvent was simultaneously evaporated at $25^{\circ} \mathrm{C}$ under different ambient pressure, which were $760 \mathrm{mmHg}$ or so-called ' normal rate of solvent evaporation' (NRSE) process, 460 and $160 \mathrm{mmHg}$ (or FRSE) process, several hours for solvent evaporation. After 2 and $6 \mathrm{~h}$ of solvent evaporation for FRSE and NRSE process, respectively, solidified PLLA or PDLLA microspheres were formed. The hardened microspheres were rinsed with distilled water several times and then dried at room temperature about 2 days. They were further dried in an oven for several hours at $40^{\circ} \mathrm{C}$ to remove residual solvent.

\subsection{Interfacial tension of $D C M / P V A$ solution, thermal analysis, particle sizes and surface morphology of microspheres}

The interfacial tension between DCM solution contained $1-10 \%$ of PLLA or PDLLA and $0.05 \%$ of PVA aqueous solution was measured by a face-surface tensiometer (CBVP-A3, Kyowa Interface Science Co., Tokyo, Japan) at $25^{\circ} \mathrm{C}$. 
Thermal analysis of the glass transition temperature for the microsphere samples was carried out with a differential scanning calorimeter (DSC 910S, Du-Pont Corp., USA). The samples (5 mg) before and after hydrolysis were scanned in aluminum pans at a heating rates of $5^{\circ} \mathrm{C} / \mathrm{min}$ to produce thermographs over the range $40-$ $180^{\circ} \mathrm{C}$. The DSC thermograph shown on the study was the second heated histogram as other investigators' study (Hausberger and Deluca, 1995).

The particle sizes of lidocaine/PLLA and lidocaine/PDLLA microspheres were determined by a laser particle analyzer (Galai CIS counter, USA) after being suspended in $10 \mathrm{ml}$ of 0.05 wt. $\%$ of sodium dodecyl sulfate (SDS) solution.

The morphological difference of the surface of lidocaine/PLLA or lidocaine/PDLLA microspheres prepared with FRSE or NRSE process was examined by a scanning electron microscopy (SEM, Hitachi S800, Tokyo, Japan).

\subsection{Drug content and release studies of microspheres in vitro}

The microspheres were firstly dissolved in DCM and drug contents of microspheres were then determined by extraction of lidocaine with $0.1 \mathrm{~N} \mathrm{H}_{2} \mathrm{SO}_{4}$ solution. In general, $1.5 \mathrm{mg}$ of each batch of the PLLA or PDLLA microspheres was dissolved in DCM, and then $5 \mathrm{ml}$ of $0.1 \mathrm{~N} \mathrm{H}_{2} \mathrm{SO}_{4}$ solution was poured into the above solution for the extraction of lidocaine. After mixed with a vortex mixer, they were centrifuged at $900 \times g$, and the upper level of solution was pipetted out for analyzing by a double beam of $\mathrm{UV} /$ vis spectrophotometer at wavelength of 214 $\mathrm{nm}$ (Jasco UV/vis 530, Kobe, Japan, Huang et al., 1997, 1999). The results of lidocaine analysis in this study were verified and consistent with those obtained by capillary electrophoresis in our earlier studies (Huang et al., 1997, 1999). The encapsulation percentage of the microspheres was determined by the ratio of actual contents of drug/theoretical maximum load of drug.

To study the releases of drug from micro- spheres, $1.5 \mathrm{mg}$ of lidocaine loaded microspheres were suspended in $1 \mathrm{ml}$ of $25 \mathrm{mM}$ of isotonic

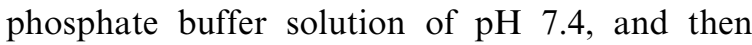
placed within a osmosis membrane with cut off molecular weight of $1 \mathrm{kDa}$ (Spectrum medical industries Inc., USA). The samples within the membrane were kept in vials containing $30 \mathrm{ml}$ of PBS with pH of 7.4 and 0.1 wt. $\%$ of sodium azide as a dissolution medium, and the vials were shaken with rates of $60 \mathrm{rpm}$ at $25^{\circ} \mathrm{C}$. Dissolution medium $(1.5 \mathrm{ml})$ was periodically drawn out from the vials for analyzing lidocaine concentrations by the above-mentioned spectrophotometric method. The same volume of fresh medium was simultaneously replaced to the vials.

\section{Results and discussion}

The rate of solvent removal in hardening process of emulsified droplets in preparing PLLA and PLGA microspheres would be an important factor to affect crystalline or other characteristics of the microspheres (Sato et al., 1988; Arshady, 1991; Izumikawa, 1991; Li et al., 1996; Jeyanthi et al., 1997). For example, in preparing peptide loaded PLGA microspheres, the differences in surface morphology and porosity of core area were observed when temperature gradient or dilution of continuous phase technique was applied to remove solvent ( $\mathrm{Li}$ et al., 1996; Jeyanthi et al., 1997; Yang et al., 2000). However, in case of preparing drug loaded PLLA or PDLLA microspheres by emulsion-solvent evaporation method, the effects of FRSE and NRSE process by reducing ambient pressure on characteristics of microspheres have not been reported. We thereby studied the issue at a fixed temperature.

Smooth surface of drug free or loaded PLLA microspheres was observed when FRSE process was applied to remove solvent for forming microspheres (Fig. 1a and b). In contrast, high porosity or many pinholes in the surface of the microspheres were shown while NRSE process was applied to form microspheres (Fig. 1c and d). The results for PLLA microspheres observed in those two process were similar to others study although 

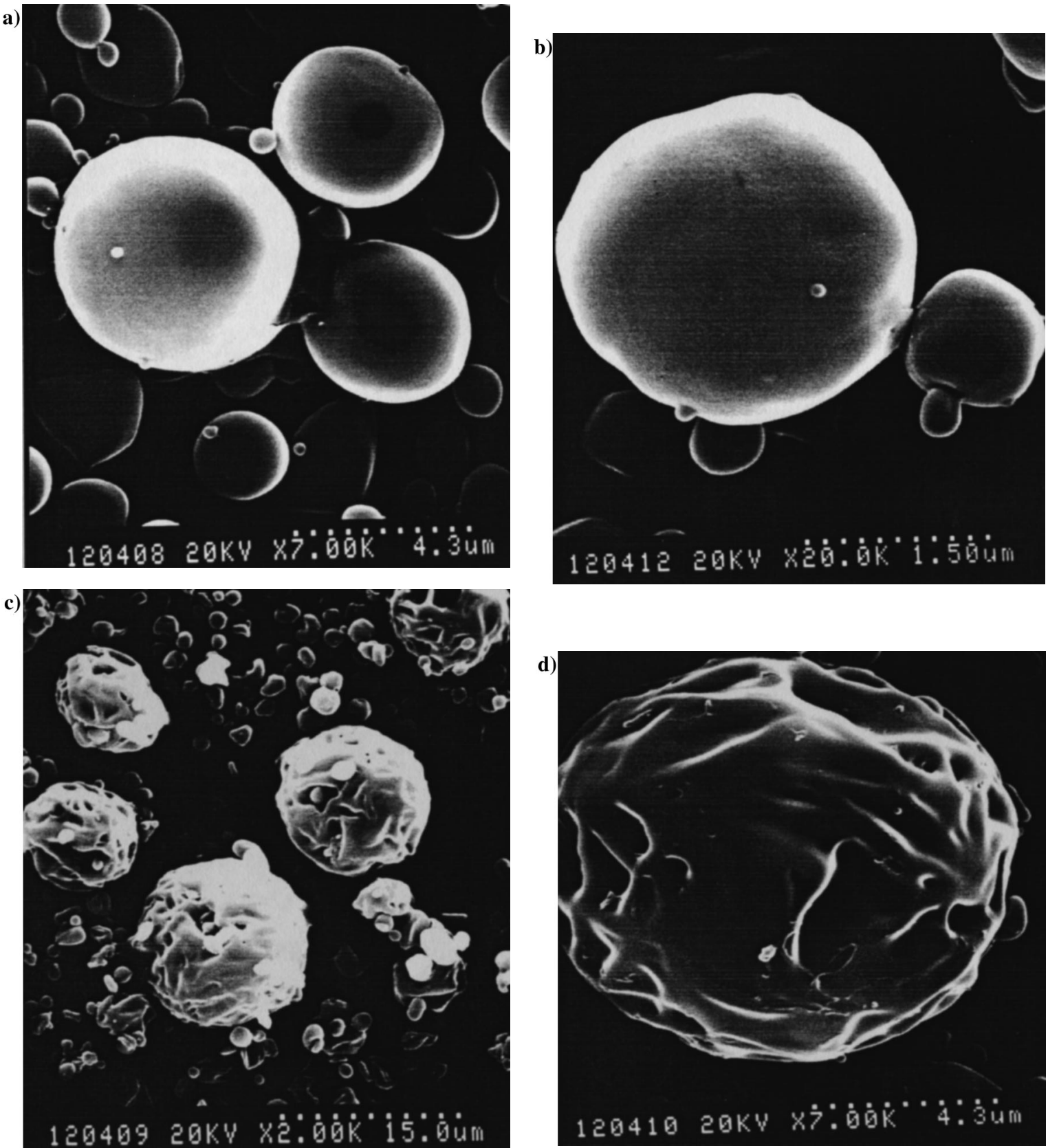

Fig. 1. (a) SEM photograph for drug free PLLA microspheres prepared with FRSE process (the ambient pressure, $160 \mathrm{mmHg}$ ). (b) SEM photograph for drug loaded PLLA microspheres prepared with FRSE process (the ambient pressure, $160 \mathrm{mmHg}$ ). (c) SEM photograph for drug loaded PLLA microspheres prepared with NRSE process. (d) SEM photograph for drug loaded PLLA microspheres prepared with NRSE process. (e) SEM photograph for drug loaded PDLLA microspheres prepared with FRSE process (the ambient pressure, $160 \mathrm{mmHg}$ ). (f) SEM photograph for drug loaded PDLLA microspheres prepared with NRSE process. 



Fig. 1. (Continued)

different in loading drug and the droplet forming method between two studies (Izumikawa, 1991).

It was interesting to find that the smooth surface of PDLLA microspheres was formed and not influenced by the FRSE and NRSE process (Fig. $1 \mathrm{e}$ and $\mathrm{f}$ ). However, smooth surface was not found in preparing peptide loaded PDLLA microspheres when FRSE process was done by increasing input rate of continuous phase (Yang et al., 2000). The differences in morphology of PDLLA microspheres observed between two studies might be due to different solvent removing techniques being applied and the hydrophobicity of drug loaded, respectively. In regarding to two materials, the different results in surface morphology between PLLA and PDLLA microspheres resulted from those two processes might be due to crystalline and amorphous characteristics for $\mathrm{L}$ and D,L type of PLA materials, respectively.

FRSE and NRSE processes also affected the particle size profiles of drug loaded PLLA and PDLLA microspheres. Both drug loaded PLLA and PDLLA microspheres prepared with FRSE process showed smaller sizes than those micro- spheres prepared with NRSE process (Fig. 2). For examples, the mean particle size for drug loaded PLLA microspheres changed from 1.14 to 0.75



Fig. 2. Particle Sizes of PLLA and PDLLA microspheres prepared with NRSE and FRSE processes $(N=3$, data presented in mean \pm S.D.). 
$\mu \mathrm{m}$ when ambient pressure was set from 760 to $160 \mathrm{mmHg}$ in solvent evaporation period (i.e. NRSE and FRSE process, respectively). Furthermore, the differences in particle size distribution of PLLA and PDLLA microspheres between FRSE and NRSE process were found before dried procedures. It has been reported that the particle sizes of PDLLA microspheres were affected by the disperse phase viscosity, stirring rate and concentration of PVA emulsifier in the emulsion process (Grandfils et al., 1992). In here, reducing the ambient pressure to enhance solvent evaporation during hardening process of emulsified droplets would also be one of the factors. One of possible explanations for smaller size of PLLA or PDLLA microspheres yielded in FRSE process might be interpreted by the Laplace equation, that could be expressed as, $d P=2 \gamma / r$ when a liquid droplet was formed in stable (Atkins, 1998), where $d P$ is the pressure difference between outside and inside of liquid film of a droplet, and $\gamma$ is the surface tension of interfacial liquid of the emulsion system, and $r$ is the radius of a spherical droplet. To estimate the possible changes of $\gamma$ value during solvent evaporation process, we measured the interfacial tension between the solutions of different weight ratio of PLLA or PDLLA dissolved in DCM and the solution of $0.05 \mathrm{wt} . \%$ of PVA at $25^{\circ} \mathrm{C}$. The interfacial surface tension was -24.0 and $-27.3 \mathrm{Nm} / \mathrm{m}$ for $10 \%$ of PLLA/DCM and $10 \%$ of PDLLA/DCM in PVA solution system, respectively. In addition, the interfacial surface tensions, $\gamma$ of the Laplace equation, of abovementioned systems were only weakly affected (e.g. less than $8 \%$ ) while varying the weight ratios (e.g. more than ten times of differences) of PLLA and PDLLA dissolved in DCM. The $\gamma$ values for the above-mentioned systems could be assumed as constants. On the other hand, the pressure differences between outside PVA solution and inside the film of PLLA or PDLLA droplets during solvent evaporation period, $d P$ of the Laplace equation, would be larger for FRSE process compared with that for NRSE process. According to the Laplace equation, we could qualitatively estimate that the mean radius of microspheres yielded with FRSE process should be smaller than that for NRSE process for forming a stabilized liquid droplet.



Fig. 3. Lidocaine encapsulation efficiency of PLLA and PDLLA microspheres prepared with NRSE and FRSE process $(N=3$, data presented in mean \pm S.D. $)$.

The recovery of lidocaine for PLLA and PDLLA microspheres prepared with NRSE process were 70.6 and $28.8 \%$, respectively. However, there were only 45.6 and $20.7 \%$ of lidocaine recovered for PLLA and PDLLA microspheres prepared with FRSE process (Fig. 3), respectively. It showed that microspheres prepared with NRSE process had higher encapsulation efficiency than those prepared with FRSE process for both PLA materials. Whereas in Izumikawa's study, the percentage of progesterone encapsulated into PLLA microspheres was higher at solvent removal under reduced pressure than that under atmosphere pressure (Izumikawa, 1991). The different results between two studies might be due to different droplet forming methods. In this study, we applied $\mathrm{O} / \mathrm{W}$ emulsification process to form PLLA droplets in stead of mechanical stirred method reported by theirs. The $\mathrm{O} / \mathrm{W}$ emulsifying process produced both PLLA and PDLLA microspheres with the particle sizes about or less than $1 \mu \mathrm{m}$ compared with $\sim 50 \mu \mathrm{m}$ for mechanical stirred method (Izumikawa, 1991).

To interpret higher in lidocaine, a crystalline drug, encapsulated into microspheres prepared in NRSE process, we proposed that higher crystalline structure of the PLLA microspheres prepared with this process (shown on DSC 
thermograph; Fig. 5) would be an important factor. The other factor might be due to large surface area for PLLA microspheres prepared with NRSE process because of high porosity or more pinholes within the microspheres (Fig. 1c and d). Moreover, more hydrophobic and higher crystalline characteristics of PLLA polymer matrix than that of PDLLA (Sah et al., 1994) would be an important factor to contribute higher efficiency of lidocaine, a hydrophobic and crystalline anaesthetic, encapsulated into PLLA microspheres than that of PDLLA ones prepared with whatever process. We noted that the match of hydrophobicity between drug and polymer matrix did affect drug encapsulation efficiency when we loaded lidocaine and propranolol $\mathrm{HCl}$ to PLLA/PEG microspheres (Huang et al., 1997).

Initial burst releases from PLLA and PDLLA microspheres prepared in both FRSE and NRSE processes were observed although that for PLLA microspheres was slightly slower than PDLLA ones for the first few hours (e.g. 10 h, Fig. $4 \mathrm{a}$ and b). While in the subsequent stage, there was no difference in drug release rate between PLLA and PDLLA microspheres prepared from whatever process, respectively. The initial burst of drug releases for PLLA and PDLLA microspheres would be due to weak adhesion or low solubility of the drug to PLA materials. The crystalline structure and hydrophobicity of PLLA polymer matrix might also play a role on lowering drug burst release from its microsphere compared with that from PDLLA microsphere (Fig. 4a and b). To reduce the initial burst of drug releases of PLLA or PDLLA microspheres, post-coated the microspheres with gelatin (Huang et al., 1999) or other methods such as multiple wall process (Pekarek et al., 1994) might be effective.

After the initial stage, pinhole structures of the PLLA microspheres prepared with NRSE process might be easily diffused by PBS buffer into inner side of spheres but the crystallization structure of PLLA microspheres after hydration might counteract the morphological disadvantage in drug releases. It was therefore that the PLLA microspheres prepared by both processes showed about the same rate in drug release (Fig. 4a) in the subsequent stage. The observations could be inferred from DSC thermographs which showed that PLLA microspheres prepared with NRSE process increased their crystallized structure after 2 days of hydration whereas losing their crystal-
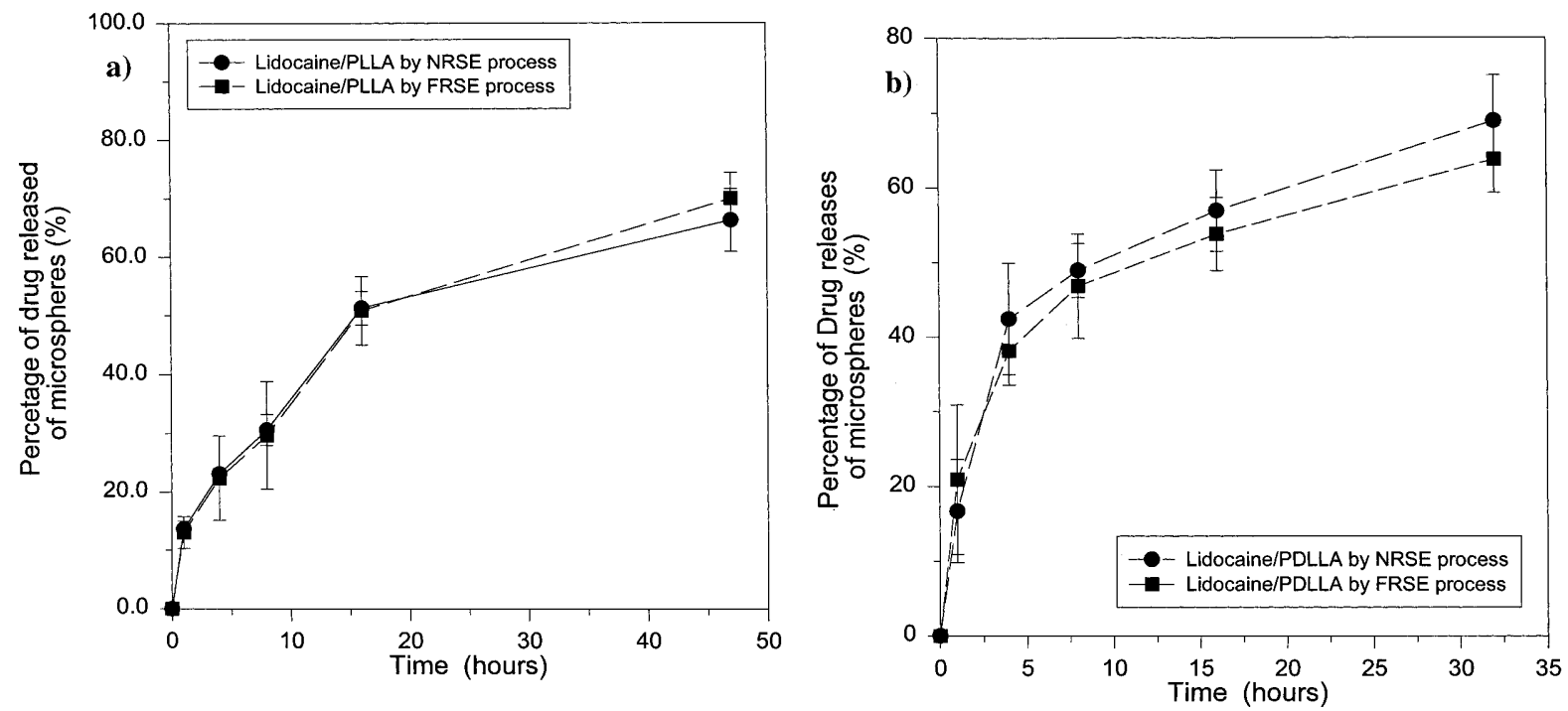

Fig. 4. (a) Lidocaine release from PLLA microspheres prepared with NRSE and FRSE processes $(N=3$, data presented in mean \pm S.D.). (b) Lidocaine release from PDLLA microspheres prepared with NRSE and FRSE processes $(N=3$, data presented in mean \pm S.D.). 




Fig. 5. Thermographs of PLLA microspheres before and after 2 and 4 days of hydration in PBS buffer solution.

lize structure in those prepared with the FRSE process was showed (Fig. 5).

In consistent with PLLA microspheres, PDLLA microspheres prepared with FRSE process showed slower releases of drug compared with those prepared with NRSE process after the burst stage (Fig. 4b). Since the morphology of lidocaine loaded PDLLA microspheres prepared with FRSE and NRSE process did not show any difference, the removal rate of solvent in $\mathrm{O} / \mathrm{W}$ emulsion-solvent evaporation process in this study would probably not affect surface morphology of amorphous materials. The differences in lidocaine releases at the subsequent stage for the PDLLA microspheres between FRSE and NRSE process might be due to differences in core structure of the microspheres. The difference in core structure of PLA based microspheres resulted from different rates of solvent removal techniques has been reported by other investigators when PLGA microspheres were fabricated (Jeyanthi et al., 1996; Yang et al., 2000). The further investigation for those differences has been scheduled.
In conclusion, the solvent removal rate by reducing ambient pressure for emulsion-solvent evaporation process would affect the surface morphology of lidocaine loaded PLLA microspheres but not in PDLLA ones. The particle sizes for PLLA or PDLLA microspheres prepared with FRSE process were smaller than those prepared with NRSE process. However, solvent removal in FRSE process produced lower drug encapsulation efficiencies for both PLLA and PDLLA microspheres than that in NRSE process did. It was also noted that the encapsulation efficiency for PLLA microspheres was higher than that for PDLLA in whatever solvent removal process. Moreover, the initial burst of lidocaine release from PLLA microspheres prepared in both processes was slower than that of PDLLA ones. Through the study, controlling solvent removal rate by reducing ambient pressure during emulsion-solvent evaporation process for preparing different characteristics of PLA based microspheres could be further applications in drug delivery. 


\section{Acknowledgements}

We acknowledge that the funding of this work was partially supported by the National Science Council of Taiwan, R.O.C.

\section{References}

Arshady, R., 1991. Preparation of biodegradable microspheres and microencapsules: 2. Polylactides and related polyesters. J. Control. Release 17, 1-22.

Atkins, P.W., 1998. Physical Chemistry, sixth ed. Oxford University Press, Oxford, UK.

Atkins, T.W., Peacock, S.J., Yates, D.J., 1998. Incorporation and release of vanco-mycin from poly(D,L-lactide-co-glycolide) microspheres. J. Microencapsulation 15, 31-44.

Conti, B., Pavenetto, F., Genta, I., 1992. Use of polylactic acid for the preparation of microparticulate drug delivery system. J. Microencapsulation 19, 153-159.

Cowsar, D.R., Tice, T.R., Gilley, R.M., English, J.P., 1985. Poly(lactide-co-glycolide) microspheres. Methods Enzymol. $112,101-116$.

Esposito, E., Cortesi, R., Cervellati, F., 1997. Biodegradable microspheres for sustained delivery of tetracycline to the perioclotal pocket: formulatory and drug release studies. J. Microencapsulation 14, 175-187.

Gogolewski, S., Jovanovic, M., Perren, S.M., Dillon, J.G., Hughes, M.K., 1993. Tissue response and in vivo degradation of selected polyhydroxyacid: polylactides (PLA), poly (3-hydrobutyrate) (PHB), and poly (3-hydrobutyrate-co-3hydroxy-valerate) (PHB/VA). J. Biomed. Mater. Res. 27, 1135-1148.

Grandfils, C., Flandroy, P., Nihant, N., Barbette, S., Jerome, R., Teyssie, P., Thibaut, A., 1992. Preparation of poly $(\mathrm{D}, \mathrm{L})$ latide microspheres by emulsion-solvent evaporation, and their clinical applications as a convenient embolic materials. J. Biomed. Mater. Res. 26, 467-479.

Gupta, R.K., Alonso, M.J., Langer, R., Siber, G.R., 1997. Chronic local tissue reactions, long-term immunogenicity and immunologic priming of mice and guinea pig to tetanus toxoid encapsulated in biodegradable polymer microspheres composed of polylactide-co-glycolide polymers. Vaccine 15, 1716-1723.
Hausberger, A.G., Deluca, P.P., 1995. Characterization of biodegradabe poly(D,L-lactide-co-glycolide) polymers and microspheres. J. Pharm. Biomed. Anal. 13, 747-760.

Huang, Y.Y., Chung, T.W., Tzeng, T.W., 1997. Drug release from PLA/PEG microparticles. Int. J. Pharm. 156, 9-15.

Huang, Y.Y., Chung, T.W., Tzeng, T.W., 1999. A method using PLA/PEG for drug release with reduced initial burst. Int. J. Pharm. 182, 92-98.

Izumikawa, S., 1991. Preparation of poly(L-lactide) microspheres of different crystalline morphology and effect of crystalline morphology on drug release rate. J. Control. Release 15, 133-140.

Jeyanthi, R., Mehta, R.C., Thanoo, B.C., Deluca, P.P., 1996. Effects of solvent removal technique on the matrix characteristics of polylactide/glycolide microspheres for peptide delivery. J. Control. Release 38, 235-244.

Jeyanthi, R., Mehta, R.C., Thanoo, B.C., Deluca, P.P., 1997. Effect of processing parameters on the properties of peptide-containing PLGA microspheres. J. Microencapsulation 14, 163-174.

Le Corre, P., Le Guevello, P., Gajan, V., Chevanne, F., Le Verge, R., 1994. In vitro controlled release kinetics of local anaesthetics from poly (D,L-lactide) and poly (lactide-coglycolide) microspheres. J. Microencapsulation 14, $243-$ 255.

Li, W.I., Anderson, K.W., Mehta, R.C., Deluca, P.P., 1996. Prediction of solvent removal profile and effect on properties for peptide-loaded PLGA microspheres prepared by solvent extraction/evaporation method. J. Control. Release 37, 199-214.

Pekarek, K.J., Jacob, J.S., Mathiowitz, E., 1994. Double walled polymer microspheres for control release. Nature 367, 258-260.

Sah, H.K., Toddywala, R., Chien, Y.W., 1994. The influence of biodegradable micro-encapsule formations on controlled release of a protein. J. Control. Release 30, 201-211.

Sato, T., Kanke, M.M., Schroeder, H.G., Deluca, P.P., 1988. Porous biodegradable microspheres for controlled drug delivery. I. Assessment of processing conditions and solvent removal technique. Pharm. Res. 5, 21-30.

Yang, Y.Y., Chung, T.S., Bai, X.L., Chan, W.K., 2000. Effect of preparation conditions on morphology and release profiles of biodegradable polymeric microspheres containing protein fabricated by double-emulsion method. Chem. Eng. Sci. 55, 2223-2236. 